\title{
Understanding patterns of deprivation
}

\author{
By The Area Based Analysis Unit, Office for National Statistics ${ }^{1}$
}

\section{Abstract}

The Index of Multiple Deprivation (IMD) provides a measure of relative deprivation across England.

This article looks at how the IMD for 2007 varies across the country and between regions. It also considers the relationship between the Index and the key National Statistics classifications - focusing on how the social type of an area and its urban/rural characteristics influence deprivation. The analysis shows that the geographic extremes (inner cities and the most remote rural areas) coincide with extremes of deprivation, while suburban and 'middle-England' almost entirely escape them.

Summary advice is provided on using the indices in small area analysis. The article should be of interest to anyone wishing to understand more about deprivation either nationally or in their own area.

\section{Introduction}

This article focuses on the Index of Multiple Deprivation (IMD) and aims to provide an analysis of what it tells us about how deprivation varies across England.

The article consists of the following sections:

- An introduction to the IMD and its construction.

- A study of the distribution of the IMD across the country How do the different types of deprivation vary from place to place? How do different regions fare with respect to the different dimensions of deprivation?

- A consideration of the relationship between the IMD and National Statistics Area Classification - How useful is the 'type' of an area in explaining the types of deprivation experienced?

- A similar study of the relationship between IMD and the National Statistics Rural and Urban Area Classification - How do cities and the countryside fare in the IMD?

The analysis shows that the scale at which deprivation is studied has a strong influence on the results. It also shows that the different dimensions of deprivation vary a great deal from region to region and, at a lower scale, show very different patterns from each other.

As well as the traditionally expected concentration of deprivation in inner cities significant areas of deprivation are also revealed in the more remote parts of rural areas - while 'suburban' areas fare much better. Above all the article demonstrates the complex and multi-faceted nature of deprivation and that 'place' matters a great deal in determining how deprived an area is likely to be.

\section{Index of Multiple Deprivation 2007}

The Index of Multiple Deprivation (IMD) provides a summary measure of relative deprivation for every Local Authority District, Unitary Authority and Lower Layer Super Output Area (LSOA) in England². The IMD aims to provide a nationally 1 The main contributors to this article were Alistair Calder, Angela Medland,
Alistair Dent and Paul Allen.
2 For further information on Super Output Areas and to download data at the LSOA, Local Authority and County level for ID2004 and ID2007 see Neighbourhood Statistics website: www.neighbourhood.statistics.gov.uk 
consistent measure of how deprived an area is by identifying the degree to which people are disadvantaged by factors such as low income, unemployment, lack of education, poor health, and crime.

The most recent IMD formed part of a package of indices published in December 2007 by Communities and Local Government (CLG). The Indices are widely used to analyse patterns of deprivation, identify areas that might benefit from special initiatives or programmes and to determine eligibility for funding.

The IMD is published at the Lower Layer Super Output Area level and formed by pulling together a total of 38 individual indicators, chosen to cover a range of economic, social and housing issues for each small area in England. The majority of the data underpinning the IMD represent data for 2005 although some data are averages covering two or three years in order to improve statistical reliability.

Figure 6.1 shows how the 38 indicators (shown as circles) are pulled together to form scores for seven separate topics always described as 'domains':

- Income deprivation

- Employment deprivation

- Health deprivation and disability

- Education, skills and training deprivation

- Barriers to housing and services

- Living environment deprivation

- Crime

The way in which scores are developed varies from domain to domain. The underlying indicators which make up the indices are weighted differently and often using differing methods for each of the different domains and sub-domains. Some are simple sums of indicators; others are constructed using more complex techniques such as factor analysis. More details on the construction of individual domain scores can be obtained from the CLG website ${ }^{3}$.

Once domain scores have been established for each area the individual areas are ranked. Areas are ranked for each domain (and for the combined IMD measure - see below) according to their position relative to all others in the country - the LSOA ranked 1 being the most deprived and the LSOA ranked 32,482 , the least deprived. When considering IMD ranks low numbers are always worse than high ones.

Scores and ranks are published for all domains and, where appropriate, for the sub-domains that make them up. A number of summary measures which describe differences in the IMD between local authority districts are also available but

3 Detailed information on the construction of indices and scores can be found here: www.communities.gov.uk/documents/communities/ doc/615986.doc it is the lowest level of information available - the LSOA measures - that we focus on here.

\section{Constructing the IMD from the individual domains}

The Index of Multiple Deprivation (IMD) provides an overall measure by pulling together a weighted combination of transformed and standardised scores from each of the seven domains.

Figure 6.1 shows the relative weights (out of 100) used to combine the scores from each of the domains. The final weights applied were the result of a combination of analysis of the data, expert opinion and consultation. Income and Employment deprivation make up 45 per cent of the IMD between them and Health and Education deprivation make up a further 27 per cent. Barriers, Crime and Living Environment

\section{Figure 6.1 Index of multiple deprivation 2007: domains, sub-domains and relative weighting}

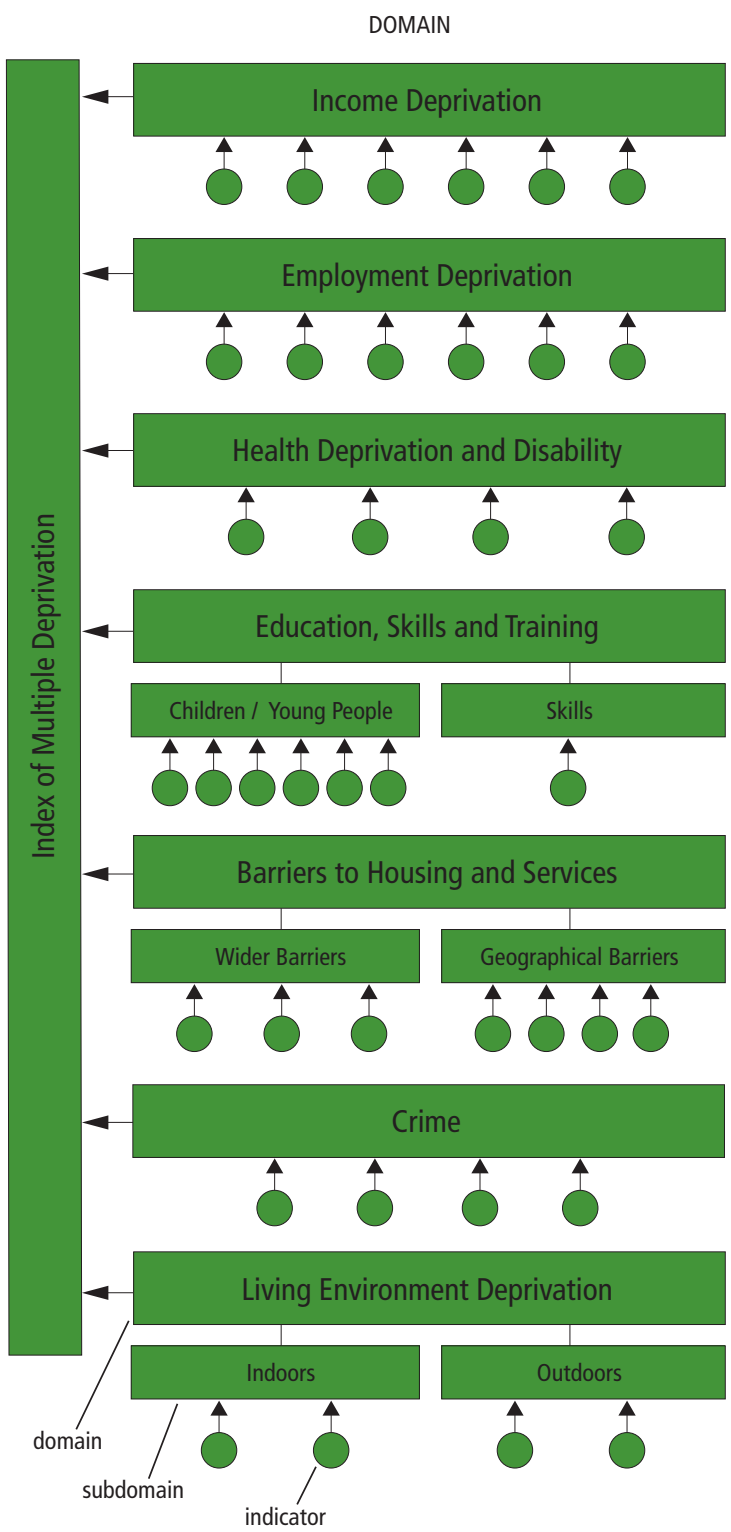

WEIGHT 
deprivation have less influence with weights of less than 10 per cent each. Income and Employment deprivation are more than twice as important as these latter domains. A later section shows how levels of deprivation identified under each of these domains vary across the country and differ from each other.

\section{Further background: The Indices of Deprivation}

The IMD forms part of a package of indicators - The Indices of Deprivation - maintained by CLG.

Sets of indicators published for Wards in 1998 and 2000 were extended to form a more complete scheme of indicators and the 'Index of Multiple Deprivation' summary measure for LSOAs in 2004.

This set of indicators (ID04) was updated, with some additions, in 2007 (ID07). While the early measures had been published at the ward level, ID04 and ID07 were published for the new Super Output Area geography which is 'frozen' and does not change over time. This, combined with the fact that the methodology underpinning ID04 and the ID07 are largely the same mean that, with some caveats, comparison between the indices is possible.

A guidance document describing the purpose, methodology and sources for construction of the indices 'The English Indices of Deprivation 2007' is available for download from the Communities and Local Government website. This document also includes an analysis of the most and least deprived 20 per cent of LSOAs on a regional basis and excellent maps of the overall IMD measure at the LSOA level. This article does not repeat this analysis - rather it provides a more detailed regional level analysis and looks within the individual domains making up the IMD.

\section{Guidance: Using the Indices of Deprivation}

The Indices are an immensely powerful tool for summarising the level of deprivation in an area or for pinpointing pockets of deprivation. They have been widely used to help focus and monitor renewal policy and are the best measures currently available for England.

There are however a number of caveats to be aware of when using the IoD to analyse small areas.

- Most importantly it is critical to remember that not all deprived people live in deprived areas and conversely, not everyone living in a deprived area is deprived; the indicators identify areas with characteristics associated with deprivation - not deprived people

- The Income domain (for example), does not measure income. Its main inputs are measures of the number of people on particular benefits in each area (and so assumed deprivation in terms of income). Accordingly the indices should not be used as a measure of affluence. Low levels of income deprivation do not necessarily mean an area is affluent

- The indices provide a relative measure of deprivation and therefore cannot be used to determine how much more deprived one LSOA is than another. For example, it is not possible to say that an LSOA ranked 10 is twice as deprived as an LSOA ranked 20. However, it is possible to say that the first LSOA is more deprived than the second
- The scores and ranks cannot be used as absolute measures of deprivation or to identify absolute change over time. The indices are good for spotting where things have improved (or otherwise) but some care must be taken in assessing change between ID04 and ID07. See the CLG guidance for more details

- The Income and Employment domain scores are rates. It is therefore appropriate to describe the LSOA in terms of the proportion of the population experiencing income or employment deprivation. For example, if the LSOA has a score of 0.63 on either of these domains, then we can say that 63 percent of its population is either income or employment deprived. This does not apply to the other five domains

- It is not appropriate to use the scores to compare an LSOA between domains. This is because the scores have different ranges and different minimum and maximum values. If you want to compare across domains, then ranks should always be used

- The Indices are for England only. Although the Indices for Scotland, Wales and Northern Ireland use similar methodologies, it is not valid to compare them. The Office for National Statistics is currently preparing guidance on how to approach comparison between the separate schemes 


\section{Spatial Patterns of the Indices of Deprivation}

The first stage in our analysis is to look at what the indices tell us about the pattern of deprivation across England. First we consider the overall patterns of deprivation at the LA and LSOA level, then we consider how the individual domains of the IMD vary across the country.

\section{The IMD at the Local Authority Level}

Map 6.2 shows the overall IMD rank for each local authority in England. The rank is calculated from a weighted average of the individual LSOA scores within each local authority. A rank of 1 represents the most deprived local authority and a rank of 354 represents the least deprived. Areas are shaded on the map using four equally sized groups, so that 25 per cent of local authorities fall into each group. For example, the LAs shaded dark green are the least deprived 25 per cent of local authorities.

The patterns here would seem to be fairly strong.

The least deprived areas spread across a broad swathe of the south of England and also in a block covering parts of Cumbria, Lancashire and Yorkshire. Further groups are seen in Cheshire and on the Norfolk/Suffolk coast.

The most deprived areas would seem to be focused in two distinct types of areas. Firstly there is a very clear concentration of deprived LAs in urban areas. The pattern is most noticeable around London and in a broad band that runs all the way from Liverpool to Leeds \& Sheffield and down into Nottingham. Nonetheless these areas are not unique - almost all urban LAs fall into the bottom 25 per cent of the IMD at this level. Birmingham, Plymouth, Hull, Bristol, Brighton and Newcastle all emerge as deprived.

There is, however, a second pattern here which, ironically, seems to relate to remoteness from urban areas. Coastal areas around the Wash, in Cumbria and in the South West all have LAs with high levels of deprivation according to the IMD. On inspection of this map alone there would seem to be a strong relationship between the urban / rural character of areas and their level of deprivation. Urban areas and the most remote areas would seem to be the most deprived.

\section{The IMD at the Lower Layer SOA level}

As ever, viewing the data at a lower level provides a different perspective and shows that different patterns emerge at different scales.

Map 6.3 shows IMD rankings for every Lower Layer SOA (LSOA) in England.

As would be expected, the overall pattern is very similar to that for local authorities.

The least deprived (dark green) areas are areas outside the cities but relatively close to them. A broad area of the South East along with the 'suburban' areas near many of the main cities across the whole of the country all fall into this category.
Map 6.13, later in this article, shows the location of urban areas and may be useful for reference here.

The most deprived areas (dark grey) also mirror the earlier pattern - they are very heavily focused on urban areas. LSOAs in cities are not universally highly deprived however. Areas in the least deprived group are virtually absent from cities but the severely deprived areas are fragmented - all urban areas show holes where there are 'middling' areas and all cities include pockets of low deprivation.

The 'remoteness' element of deprivation that we identified at the LA level is also clearly evident at the LSOA scale. The coastline around the Wash, the more remote part of Northumberland and Cumbria, the Welsh borders and, most strikingly, much of the South West all suffer deprivation that would appear to be directly related to their remoteness. Even coastal areas in Kent appear to be deprived in this way.

Again a comparison with the rural/urban map (Map 6.13) is worthwhile - showing what seems to be a remarkably strong relationship between the most remote rural areas and deprivation.

The extremely deprived areas (dark grey) in remote areas we identified at the LA level are less obvious at the LSOA scale but the relationship is still there. For the most part the most deprived LSOAs do seem to be 'coastal' rather than principally remote. The coast of Lincolnshire, East and West Sussex and Devon all have highly deprived coastal areas apparently related to the location of deprived coastal towns. Three additional areas are interesting - the extreme tip of Cornwall, the Isle of Sheppey on the north coast of Kent and a broad area across the middle of the Fens in the north of Cambridgeshire. All appear as highly deprived - again being 'cut-off' seems to be influencing the level of deprivation.

Remember that some of these coastal areas are likely to have a relatively elderly population and that this may be having an influence on the measured levels of deprivation. Equally it is worth noting that deprivation is a complex concept and that different things matter to different people. For some, remoteness and inaccessibility will be seen as a positive influence on their quality of life.

Whatever the precise patterns, a consideration of this data at both scales shows clearly how the LA level data smoothes out significant variations in the data. This variation clearly underlines the importance of using the lower level data wherever possible - and particularly when targeting resources.

This initial inspection of the patterns has focused on the overall IMD but it is important to remember that this is a complex and multi-faceted measure. Areas identified as deprived will all have different mixes of factors leading to their deprivation. In one area high deprivation may be related to low income or high unemployment, while in another, barriers to housing and services may be the key factor. The next section looks at how these different factors - the domains of the IMD - vary across the country. 
Map 6.2 Index of Multiple Deprivation 2007 for local authorities in England

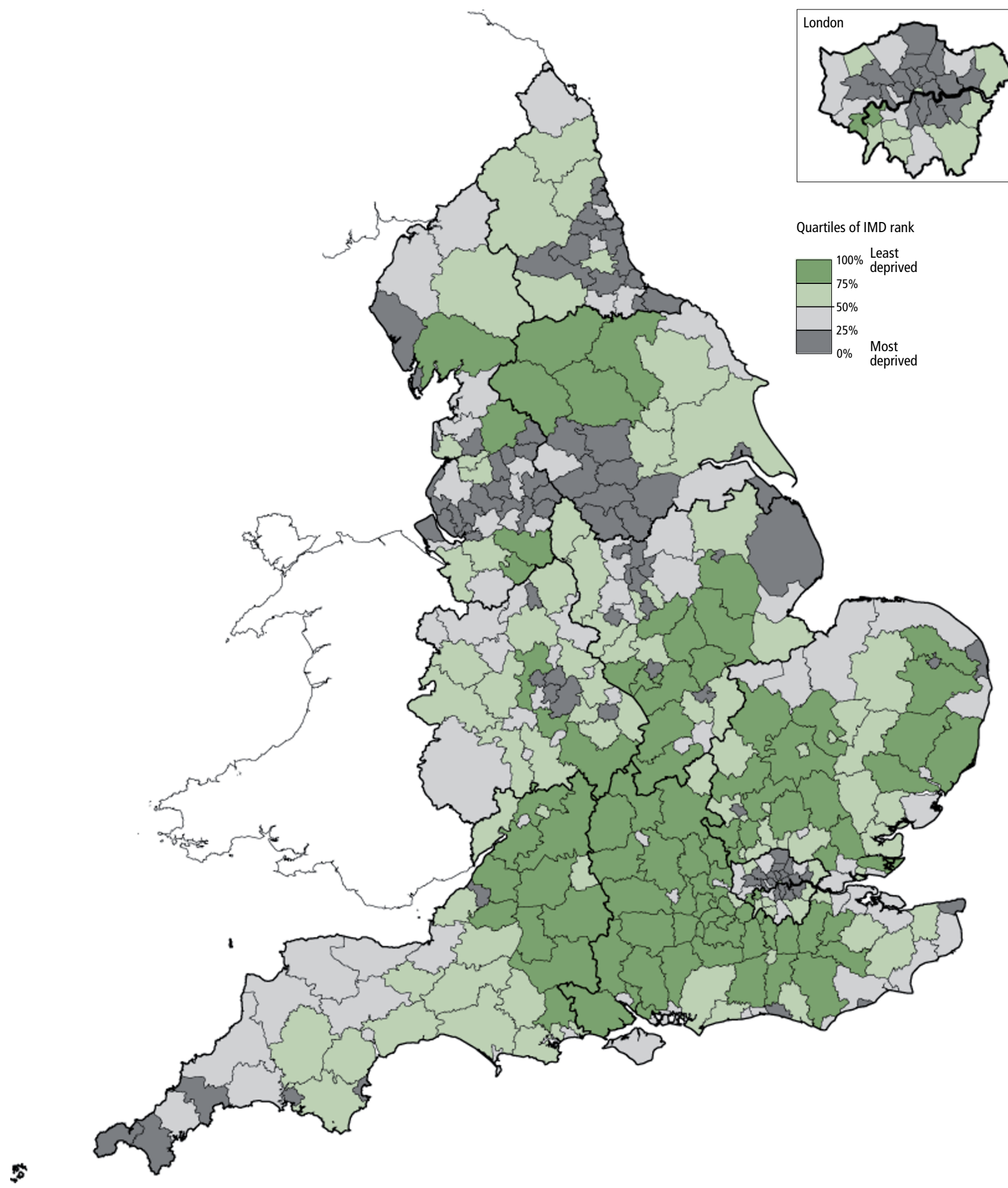

Source: Communities and Local Government 
Map 6.3 Most and least deprived LSOAs England, 2007

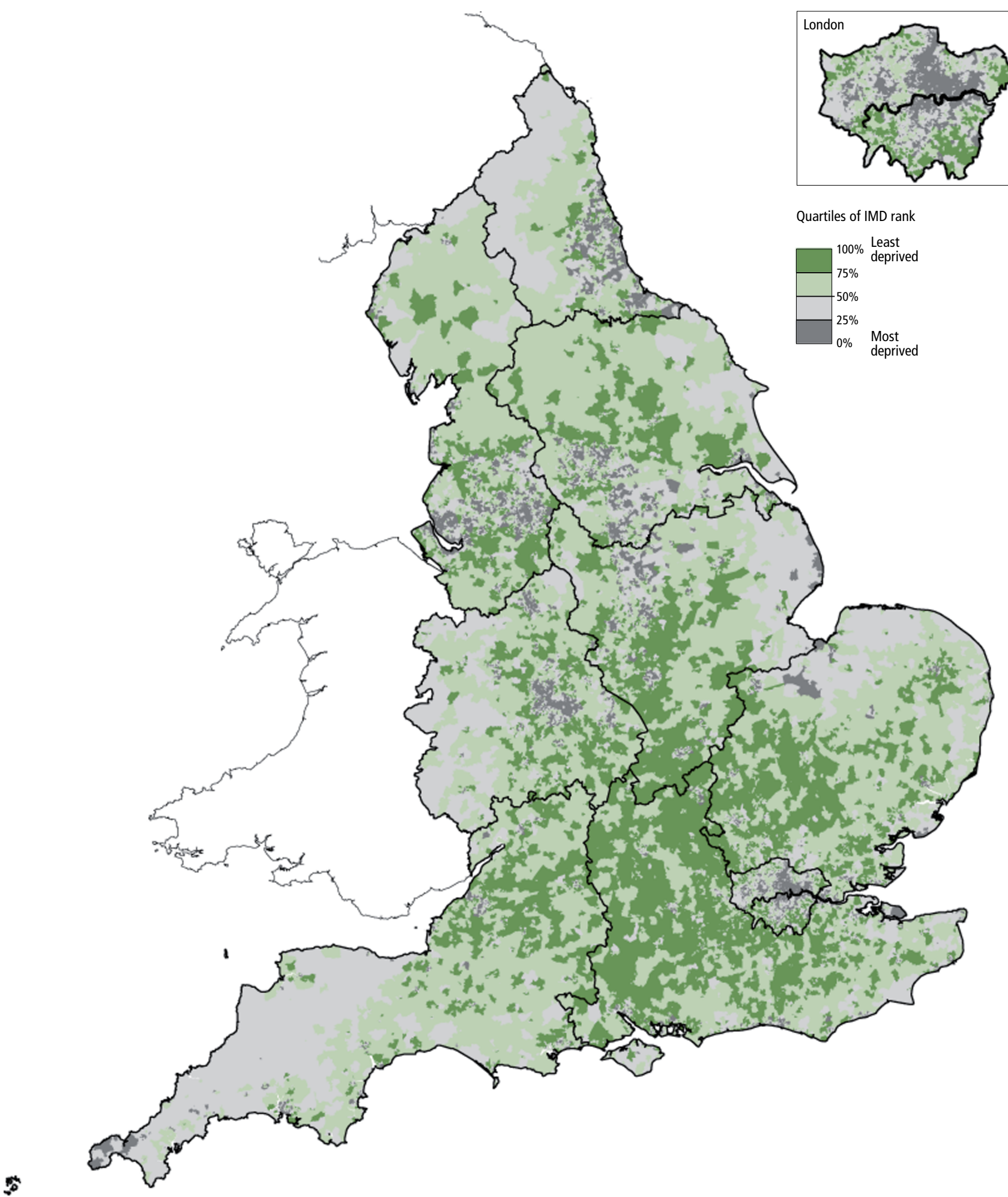

1 Lower Layer Super Output Area

Source: Communities and Local Government 


\section{Patterns for the individual domains}

The following maps show the distribution of high and low levels of deprivation for individual domains of the IMD - all at the LSOA level. For clarity at this scale only the 25 per cent most deprived (grey) and 25 per cent least deprived (green) of the SOAs are shown.

In interpreting these maps it is important to remember that our perception of these patterns is partially influenced by differences in size of the LSOAs. LSOAs are synthetic statistical areas built from postcodes and designed specifically to capture small areas of similar population size (averaging around 1,500 people). Accordingly urban LSOAs are much smaller (in area) than rural ones. The majority of LSOAs in England are 'urban' (around 80 per cent - see the later discussion) but larger suburban or rural LSOAs can seem to dominate on many of these maps. This should be borne in mind when studying the patterns.

Note: Limited space means we focus on a limited part of the country for this article - but maps plotting results for each domain for the whole of England are available to download from the Neighbourhood Statistics website.

\section{Income Deprivation}

The Income Deprivation domain aims to measure the proportion of the population experiencing income deprivation and is built using a range of measures of income related benefits. $^{4}$

Map 6.4 shows ranks for the 'Income Deprivation' domain.

There are some striking similarities to the spatial patterns shown for the combined IMD (see the darkest green areas on Map 6.3). This is perhaps not surprising as the Income domain is given one of the heaviest weightings when calculating the IMD and there is an obvious correlation between income and deprivation.

There are some subtle differences between the maps however.

Although the overall spread is very similar, a close look at the patterns in Norfolk, Suffolk and Kent for example shows that local patterns vary a great deal. Even for this domain, the most closely correlated to the IMD, different dimensions of deprivation provide subtly different results.

Interestingly a close look at the urban areas shows another difference. Although most urban areas are highly deprived both on the IMD and Income domain there would seem to be more 'non-deprived' LSOAs in the centres of some of the cities on the Income domain than the IMD. There are some pockets of relatively better off areas in cities - but these are being 'pulled-down' by other aspects of deprivation included in the IMD.

\section{Map 6.4 Income Deprivation Domain: Most and least deprived LSOAs, 2007}

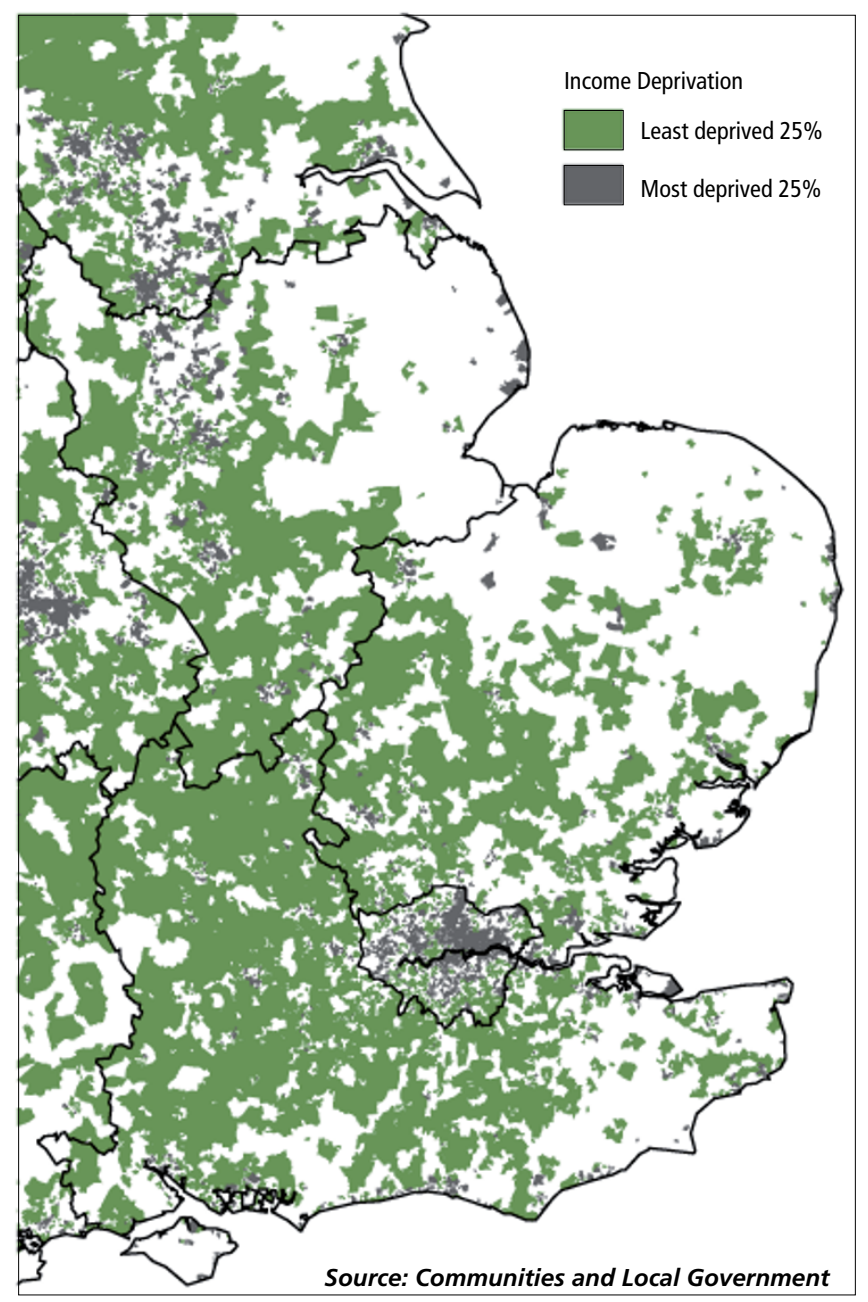




\section{Employment Deprivation}

The Employment Deprivation domain aims to measure 'involuntary exclusion of the working-age population from work' and is based on a number of measures of those receiving benefits.

Map 6.5 shows ranks for the Employment domain, and there are obvious similarities to the Income domain. However, the least deprived LSOAs for the Employment domain seem to spread further out than did the least deprived areas identified for the Income domain Map 6.4.

Examples of this can be seen in Suffolk to the North East of London and in Kent where the least deprived LSOAs for the Employment domain spread further than the least deprived LSOAs for the Income domain. London would seem to be having an influence on employment deprivation (or lack of it) far beyond its boundaries - and, interestingly, a greater effect than that observed on income.

The most deprived area around Central London also appears smaller than on previous maps suggesting that London is less employment deprived than income deprived. In the top left

\section{Map 6.5}

hand corner of the map (around Bradford/Leeds and Sheffield) the opposite is the case - this area is showing more areas with severe employment deprivation.

Remember the IMD is ranked and so a relative measure. 'Success' in one part of the country influences the rank of deprivation elsewhere.

\section{Health Deprivation and Disability}

The Health Deprivation and Disability domain identifies areas with 'relatively high rates of people who die prematurely or whose quality of life is impaired by poor health or who are disabled'.

The pattern for most and least deprived LSOAs for this domain (Map 6.6) is again broadly similar to the preceding two domains. Again, 'suburban' areas do best and cities and 'remote' areas worst.

It is difficult to be definitive from this type of analysis but, if anything, the differences between city and suburb seem to be polarised here. There would seem to be highly deprived core in the centre of London (and other cities), while deprived 'gaps' are rarer in suburban areas.

Map 6.6

\section{Health Deprivation and Disability Domain: Most and least health deprived LSOAs, 2007}

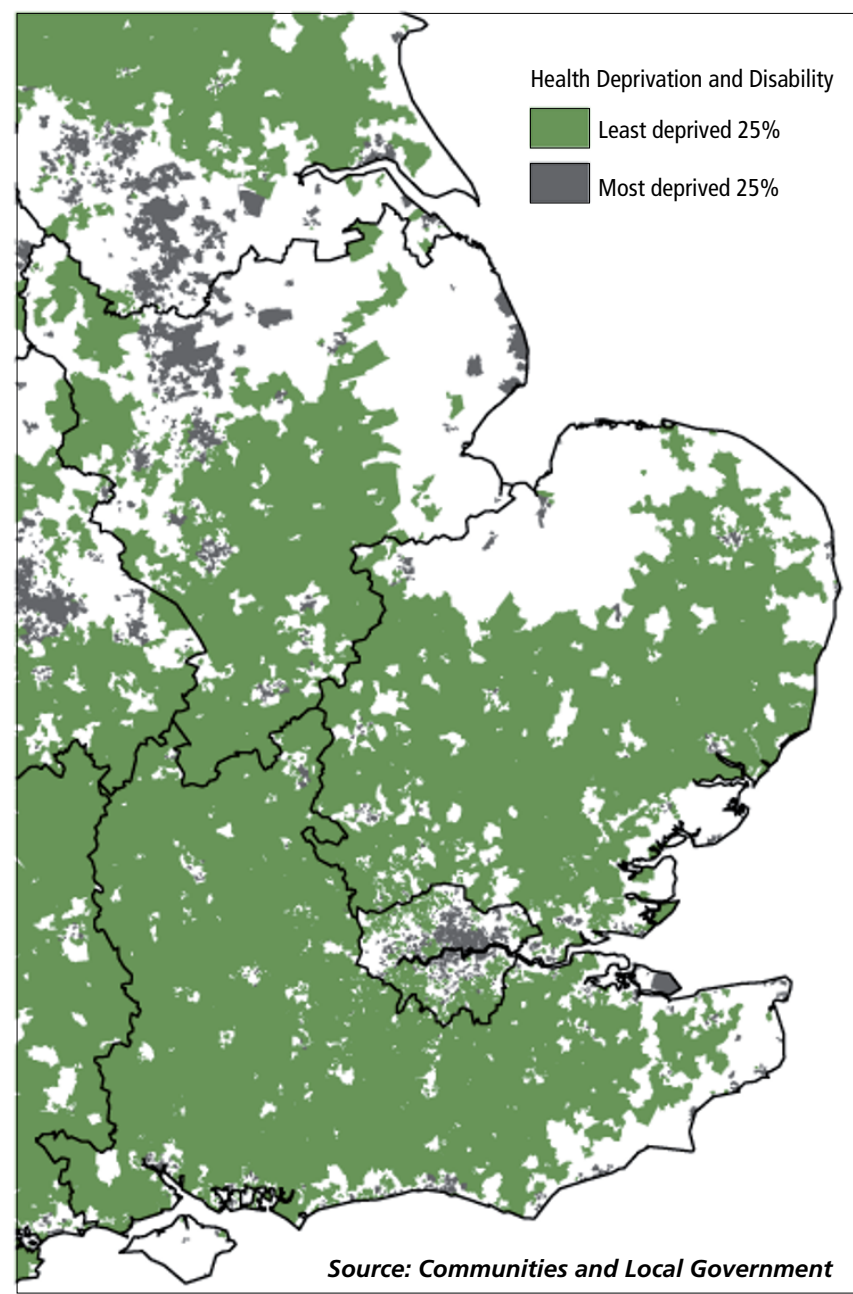


These first three domains have shown a fairly similar pattern. The remaining domains start to show more significant differences, and so to show why the IMD is such a complex measure

\section{Education, Skills and Training Deprivation}

The Education, Skills and Training Deprivation domain measures deprivation in educational attainment, skills and training using a range of Key Stage results, measures of qualifications and the number of school absences.

Again broadly non-deprived areas spread across much of the Home Counties - but on closer inspection the areas of deprivation have a quite different pattern.

The urban areas in the top left corner of the map (Leeds, Bradford, Sheffield, Nottingham) show a clear pattern of deprivation. For the most part urban areas are education deprived - but this is much less the case in London. There are, of course, severely deprived areas in London but they are more fragmented than in previous domains and there are significant areas of low deprivation within its boundary - notably to the west of the city. To some extent this is a particular London

\section{Map 6.7 Education, Skills and Training Deprivation Domain: Most and least deprived LSOAs, 2007}

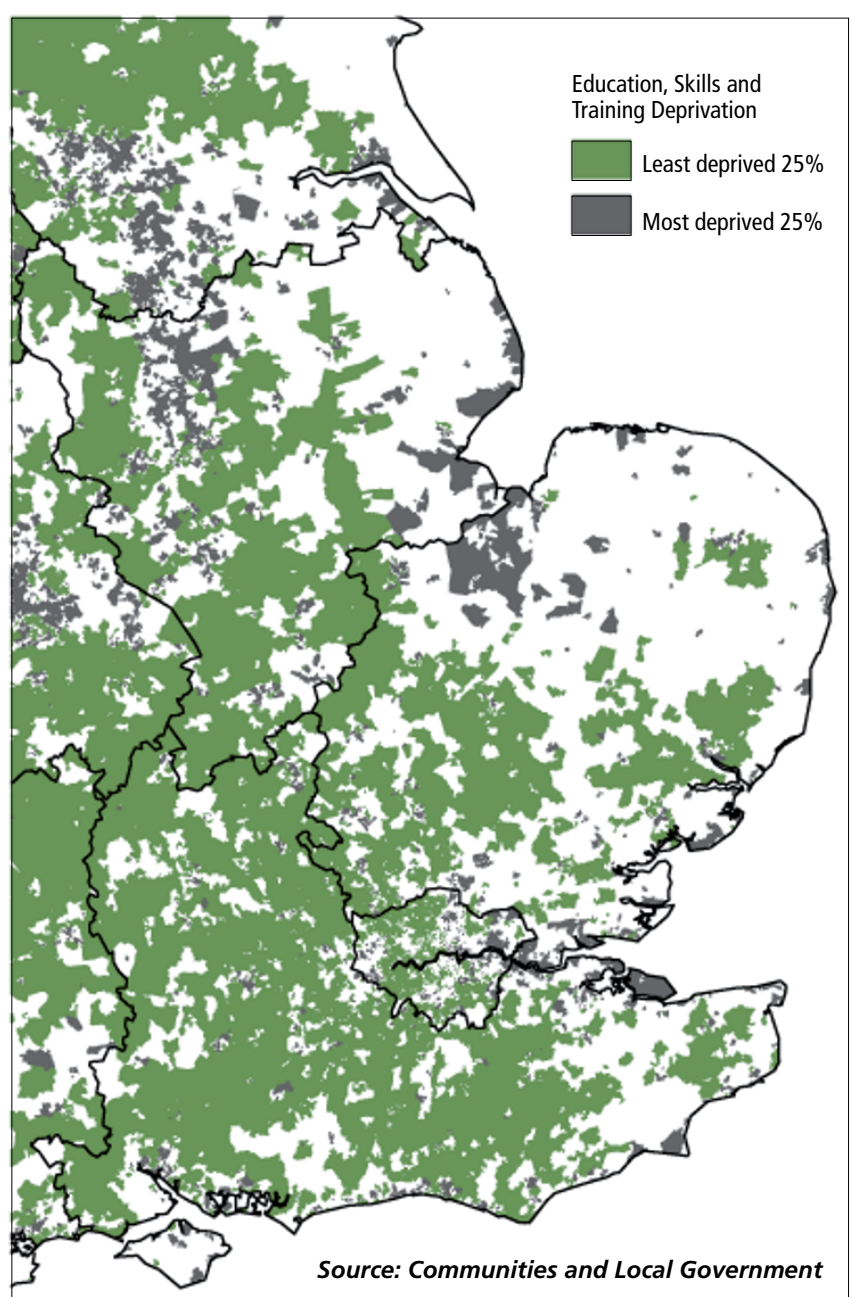

effect - London is simply less education and skills deprived than other cities. A look outside this area ${ }^{5}$ shows similar, though less marked, effects in other major cities such as Bristol, Birmingham and Manchester.

The most obvious differences here, however, are the appearance of a number of clusters of highly deprived LSOAs in the most remote areas. The most obvious of these is a group around the Fens south of the Wash but more remote parts of Essex and Kent (again including the Isle of Sheppey), and much of the coastline of Lincolnshire emerge as highly deprived. The Isle of Wight is similarly deprived. Clearly accessibility is playing some part in these areas and it seems likely that this element of deprivation may be contributing to the overall 'remoteness' effect we identified in the IMD.

\section{Crime Domain}

The Crime domain measures the rate of recorded crime for four major crime types - burglary, theft, criminal damage and violence.

5 Maps for all of England can be downloaded from the Analysis and Guidance section of the Neighbourhood Statistics website www.neighbourhood.statistics.gov.uk

Map 6.8 Crime Domain: Most and least deprived LSOAs, 2007

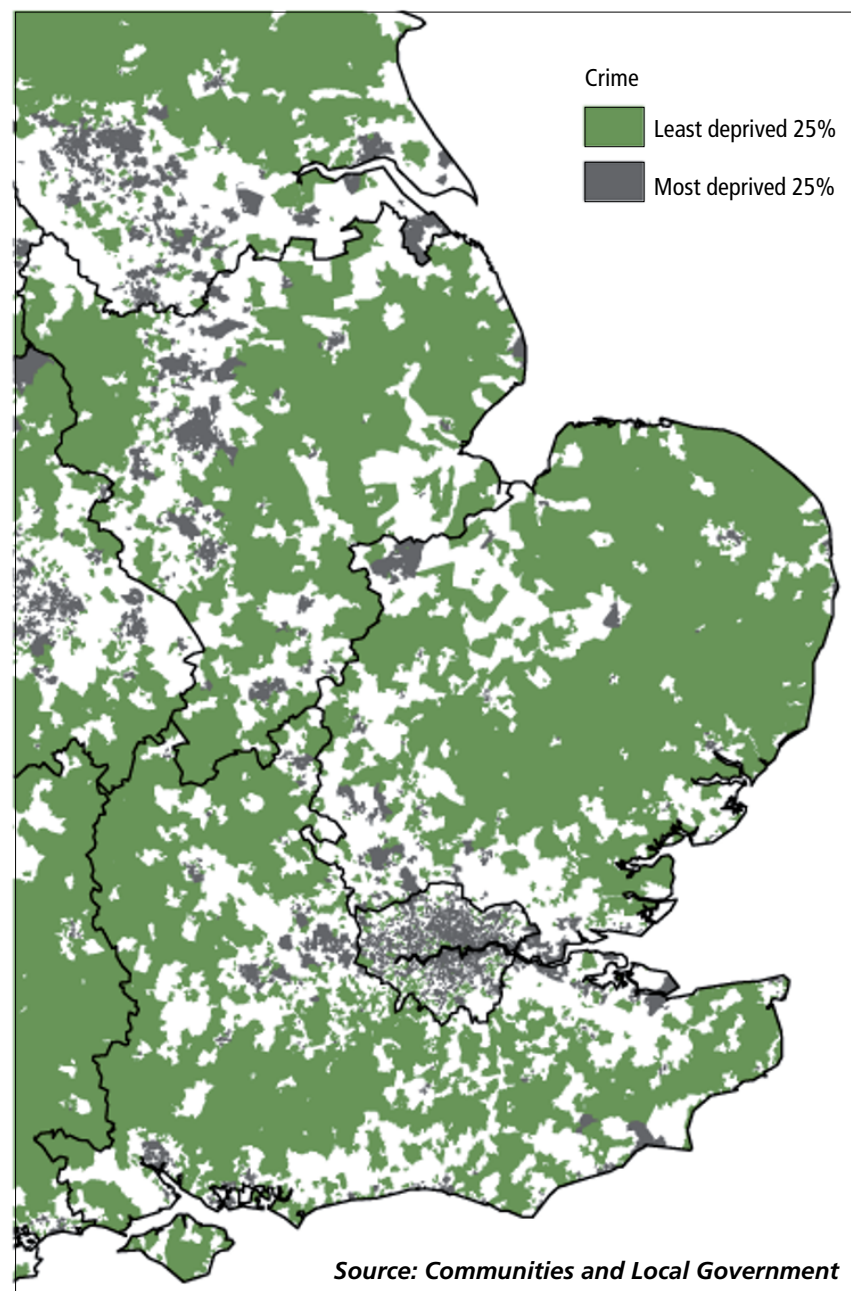


Within the Crime domain there is a very different spatial distribution. The most deprived LSOAs are mainly located in major towns and cities, while the least deprived LSOAs tend to
Map 6.9 The Living Environment Domain: Most and least deprived LSOAs, 2007

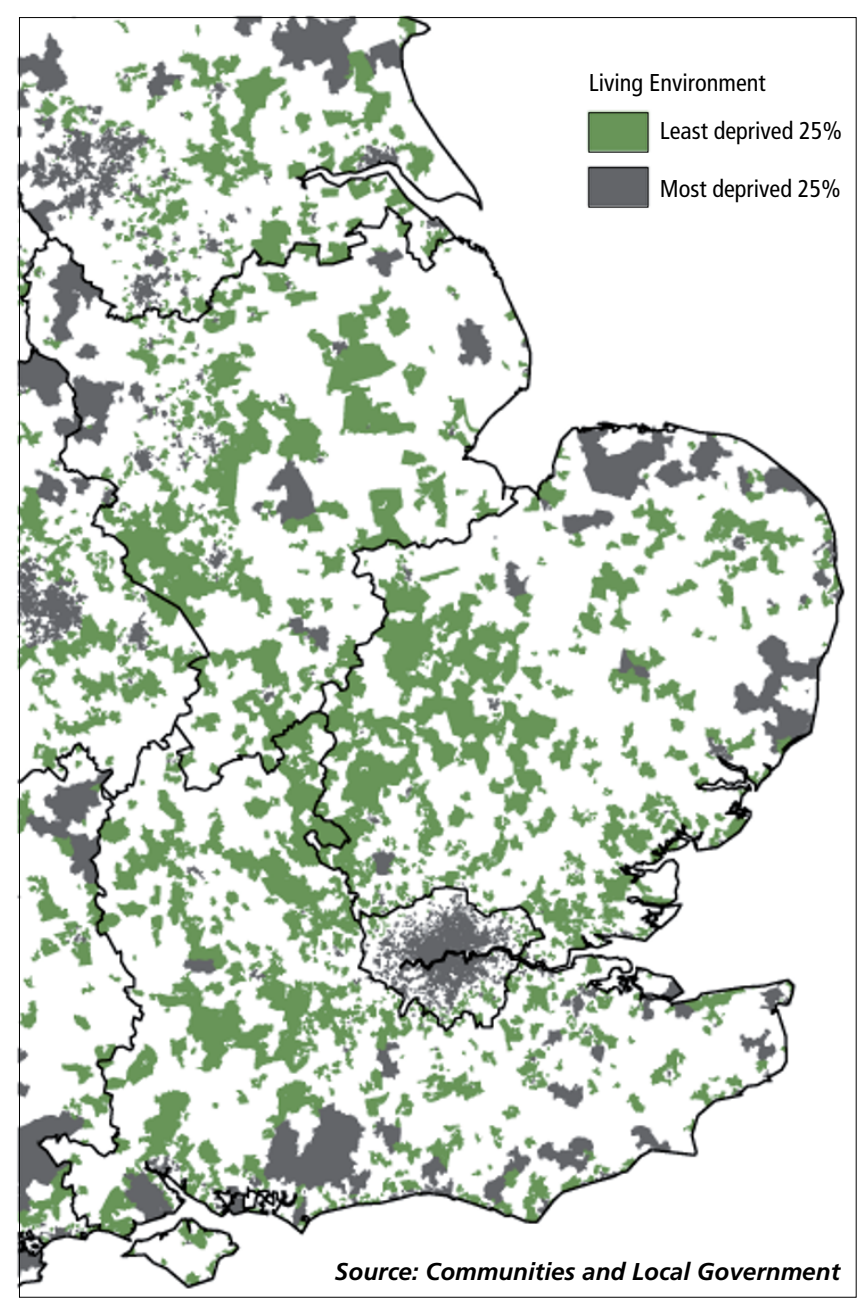

As usual the cities seem to fare badly - but, if anything, it is the very centres of urban areas that are most deprived. As well as poor housing stock these areas might be expected to suffer from poor air quality and large numbers of traffic accidents. A wider range of rural and coastal areas are also in the most deprived category - this time perhaps because of poor housing conditions. Overall the groups would seem to be polarised again in this domain.

The least deprived LSOAs are not in major cities but are scattered across the area. Better air quality, fewer accidents and a higher quality of housing stock are all likely to be contributing here. 


\section{Barriers to Housing and Services}

The Barriers to Housing and Services domain also consists of two sub-domains: 1) 'geographical barriers' which measures accessibility of shops, GPs, schools and Post Offices and 2) 'wider barriers' which includes issues relating to access to affordable housing.

Again the mixed nature of this domain results in a completely different pattern. For the first time urban areas do not dominate the most deprived areas.

Remember, as mentioned before, the maps show quartiles so each map actually has the same number of LSOAs falling in each category. The apparent 'spread' and multiplication of deprived areas here is a purely visual effect - the result of deprived areas in this case being more rural, so larger in geographic area, than previously.

The pattern here is actually close to the reverse of that on previous maps where deprivation is much more likely in rural areas. London is an exception in this domain - an urban area where increased accessibility of services is not sufficient to pull it out of deprivation, probably because of the lack of affordable housing.

\section{Map 6.10}

\section{Barriers to Housing and Services: Most and least deprived LSOAs, 2007}

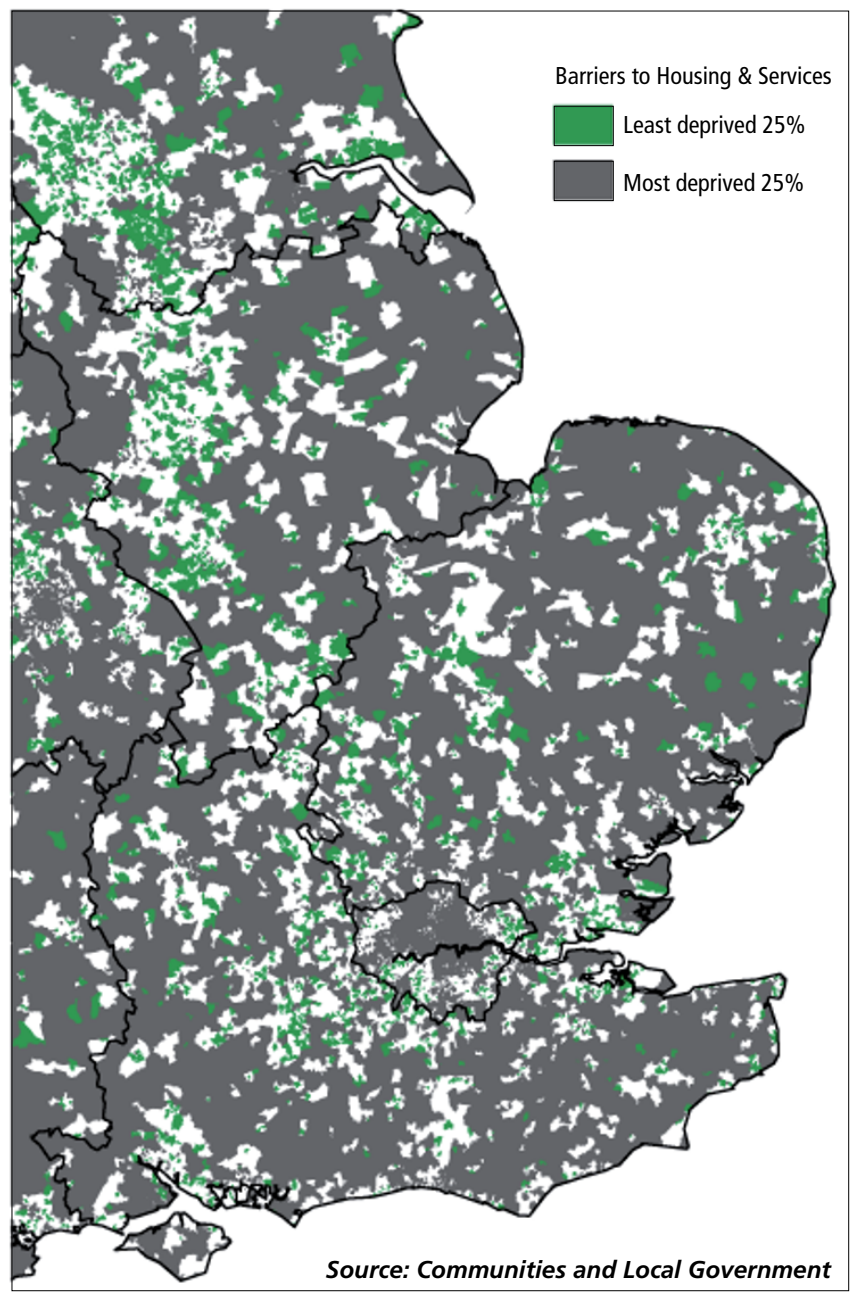

The least deprived LSOAs in this domain tend to be located near towns and cities (excluding London). All of the urban areas in the top left corner of the map have significant lessdeprived areas associated with them - the result of good access to services and more affordable housing. Interestingly the urban areas in the South East have much fewer such areas - again probably a housing cost effect.

\section{Spatial Patterns - Conclusions}

The maps above have shown that there are clear differences, as well as strong patterns of similarity, in the spatial distribution of the IMD and its separate domains.

Nonetheless, some general patterns emerge when studying the domains. There would seem to be a strong urban influence on the degree of deprivation in all of the domains but also a clear factor relating to 'remoteness' in many. The Income, Employment and Health domains display very similar patterns mirroring that for the overall IMD. Education deprivation is often urban although major cities also seem to draw in skills and an educated population - at least in places. Crime seems to have a much more direct relationship with accessibility to the urban areas than the other domains. The Living Environment and Barriers domains on the other hand show completely different patterns - in part as a result of their different and sometimes mixed (even conflicting) focuses - see later discussion.

The relationship between 'urban-ness' and different domains of deprivation are considered in more detail later.

It is clear that, particularly outside the most deprived city areas, individual LSOAs are often mixed in their level of deprivation in different domains. For example many areas will be deprived in Barriers, Living Environment or Crime domains but not in Income or Employment (and vice versa). The IMD is obviously designed to pull together these differing facets of deprivation but when carrying out small area analysis it is often worth looking closely at what the domains, and even their subdomains, tell you about different aspects of deprivation.

The analysis so far has been based on visual observation and is consequently rather subjective. The next section looks for more substantive evidence by considering the statistical distribution of the ranks and how the different regions fare on the individual IMD domains. 
Regional variations in deprivation by domain

Figure 6.11 provides regional boxplots for the IMD and each of the domains.

A box plot is a statistical graphic that provides a visual overview of a data distribution by showing the median, quartiles and spread for each area of interest. If you are unfamiliar with the use of boxplots see the Analysis and Guidance area of the Neighbourhood Statistics website, or Regional Trends 40 (Page 18) for advice.

The boxplot in the bottom left hand corner of the graphic, for example, shows the spread of results from the IMD for each region. Each of the nine bars shown represents the spread of ranks for an individual region. The box in the centre of each boxplot shows the middle two quartiles of the data - the 'interquartile range. The 'whiskers' that extend top and bottom show the full range of all ranks. ${ }^{6}$

6 Note that symbols used to indicate outliers sometimes used on boxplot are not included here and that, in this case, whiskers are used to show the whole range of observed values.
The thin black centre line running across each bar is the median value. This will differ slightly from the mean in some cases but has been chosen because it is less influenced by extreme outliers and is felt to be a better indication of the 'middle' of the range in this case.

Ranks range from 1 to 32,482 and the green line running across the centre of each boxplot indicates the middle of this range - our dividing line between more and less deprived LSOAs. Bars towards the top of the plot indicate that a region is less deprived than the national average while those at the bottom show that a region is more deprived.

The boxplot in the bottom left hand corner of the graphic is for the overall IMD ranks - and the regions are listed in order of declining median deprivation score - with the least deprived region (the South East) at the left hand side of the plot and the most deprived (the North East) at the right hand side. This same region order is used for all of the boxplots here but the 'order' of deprivation varies depending upon domain.

\section{Figure 6.11 Regional variation in deprivation - for IMD and by domain}
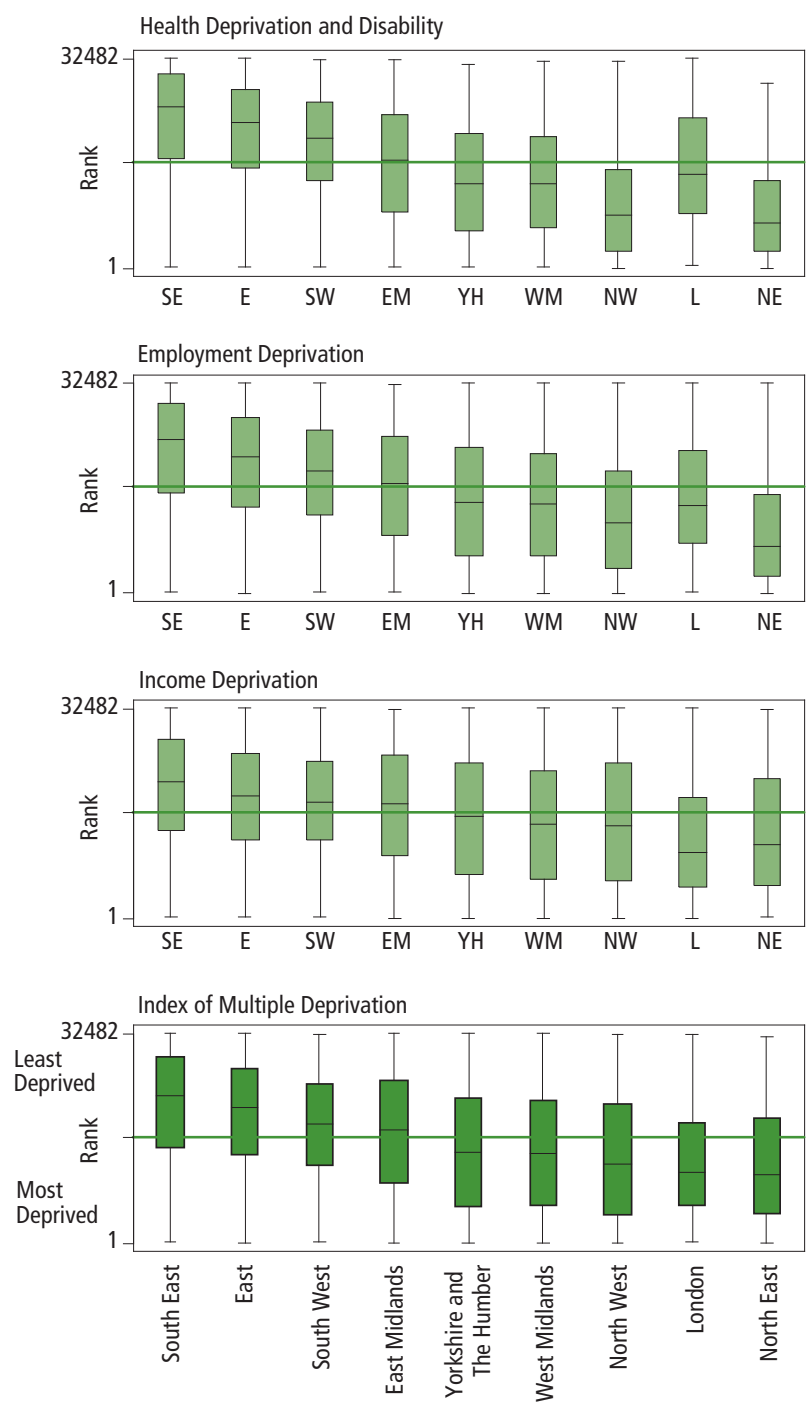

Considering first the plot for overall IMD and using the (dark green) inter-quartile boxes shown on the plot as an indicator of the distribution it is clear that there is considerable variation in the overall level of deprivation between regions.

The least deprived region here, the South East, has most of its inter-quartile range (shown by the dark green box) lying above the national mean, while the North East has almost all of its inter-quartile range lying below the mean. Other regions fall somewhere between these two and the order is interesting in its own right.

Patterns are very similar for the Income, Employment and Health domains and the order of regions very similar. In the case of Employment and Health the disparity between the least and most deprived regions seems to become even more marked - and in the case of Health all of the inter-quartile range for the South East lies above average, while the North East lies wholly below the mean.

The one region that bucks this trend is London. It performs worst of all on Income and better than its overall IMD score might suggest on Employment and Health. Remember however, as mentioned before, that the income domain is driven by benefits rather than income itself.

The Education domain, again, provides a more mixed picture at the regional level. In this case London and the South West come to rival the South East at the less deprived end of the scale while West Midlands, East Midlands, Yorkshire and the Humber and the North West are all relatively deprived - joining the North East at the bottom of the scale. The 'remoteness' element identified at the LSOA level does not seem to be significant enough to affect the results here - at least in part because most regions have some 'remote' areas.

Crime shows equally mixed patterns. Yorkshire and The Humber, the North West and London are the most deprived while the South East, East of England and particularly the South West have the lowest levels of Crime deprivation. For once the North East is seen to be less deprived than the national average. The patterns here would seem to support the earlier supposition that crime deprivation spreads around cities. The most deprived regions here all have a large part of their populations living in or near major conurbations.

The Living Environment domain is even more mixed. The most deprived region in the IMD - the North East - is the least deprived in the Living Environment domain - 80 per cent of its LSOAs lie above average. The South East, the East of England and East Midlands also fare well on this domain. Most of the other regions lie around the national average - but London is hugely disadvantaged on this measure. London's whole interquartile range lies below the average.
Finally the Barriers domain is the most mixed of all. East Midlands, Yorkshire and The Humber, the North West and the North East all do well while London, again, is very significantly the most deprived region. Regions with a large proportion of urban population would seem to be doing well - while the severe lack of affordable housing in London clearly pulls it back - 86 per cent of the LSOAs in London fall in the bottom half on this domain.

The next section of this analysis considers the relationship between the IMD domains and the National Statistics Area Classification. It attempts to answer the question: to what extent can the social characteristics of an area as summarised in the Area Classification explain and predict the different types of deprivation suffered in that area?

\section{Comparison of the Indices of Deprivation and Area Classifications}

\section{National Statistics 2001 Area Classification}

Area classifications provide a simple way of summarising the nature of different localities by grouping together areas of similar type and describing them according to their key characteristics. They can be useful in helping us focus on appropriate areas without being overwhelmed by a wide range of differences in the underlying data.

The National Statistics 2001 Area Classification of Super Output Areas and Data Zones ${ }^{7}$, which is freely available, is one such classification built from a range of 2001 Census data. Although the Area classification is based on data from 2001 and the ID07 on data from 2005 data the Classification still provides a useful way of answering the question 'What type of area is this'?

The basic technique for creating the National Statistics Area Classification is known as cluster analysis, and is well established. The cluster analysis here takes a total of 43 variables derived from Census 2001 and using this data identifies, for each area, those other areas to which it is most statistically similar. Accordingly it generates a set of statistical 'clusters' and every LSOA is allocated to one of these. Labels attached to each grouping describe the key characteristics that make up that type of area. The clusters are themselves grouped into a three-tier hierarchy referred to as Super-groups, Groups and Sub-groups.

The 'Super-group' level of the classification at LSOA level is used here to examine how different types of areas perform on the IMD07 and its constituent domains. The Area Classification Super-groups (referred to from now on as 'AC Groups') are: Countryside, Disadvantaged Urban Communities, Miscellaneous Built up Areas, Multicultural City Life, Professional City Life, Urban Fringe, and White Collar Urban. 
Analysis using the NS Area Classification: a couple of caveats

As ever it is important to remember that deprived areas are not the same as deprived people. Areas described in the National Statistics Area Classification as, for example, 'Professional City Life' do not contain only professionals. The categorisation arises from areas with certain social or statistical characteristics - not from any direct assessment of the situation of individuals. There are a range of generalisations and categorisations going on here and it is important that any conclusions drawn are used with this in mind.

Equally, it is important to note that the Area Classification used here has a direct (although relatively small) statistical relationship with the IMD. Three of the 38 indicators that make up the IMD are drawn directly from census variables - but only one of these (households with no central heating) contributed to the construction of the Area Classification. In this one case, however, exactly the same set of statistics is contributing to the clusters of the Area Classification and values the Living Environment domain.

Combination with other variables in both cases, the process of cluster analysis in the Area Classification and standardisation and ranking of scores in the case of IMD means it is virtually impossible to identify exactly how strongly this relationship holds. It seems likely that the direct link is relatively minor but it is worth being aware of.

Census data are used for denominators throughout the IMD and for the Area Classification- but this should not result in any unwanted effects in the comparison - in fact it should help in ensuring consistency of the results.

Figure 6.12 includes a set of histograms which provide a detailed view of the relationship between Area Classification classes and the IMD domains.

A careful study of the patterns here can reveal a huge amount of information and many of the subtleties of the complex relationship between IMD domains and Area Classification groups.

\section{Interpreting the histograms}

The multiple histograms included in Figure 6.12 and 6.14 are initially daunting but repay the effort required to understand them.

In the case of Figure 6.12 a separate histogram is provided for each intersection of the Area Classification and the overall IMD and its domains. Each histogram shows the count of LSOAS broken down into the deciles (10 per cent groups) of deprivation ranks and so the profile of deprivation for that domain and that type of area. LSOAs ranked in the lower deciles $\left(1^{\text {st }}-5^{\text {th }}\right)$ could be said to be 'more' deprived, with LSOAs ranked in the upper deciles $\left(6^{\text {th }}-10^{\text {th }}\right)$ being 'less' deprived.

To further explain this consider the example which is an extract from the main diagram:

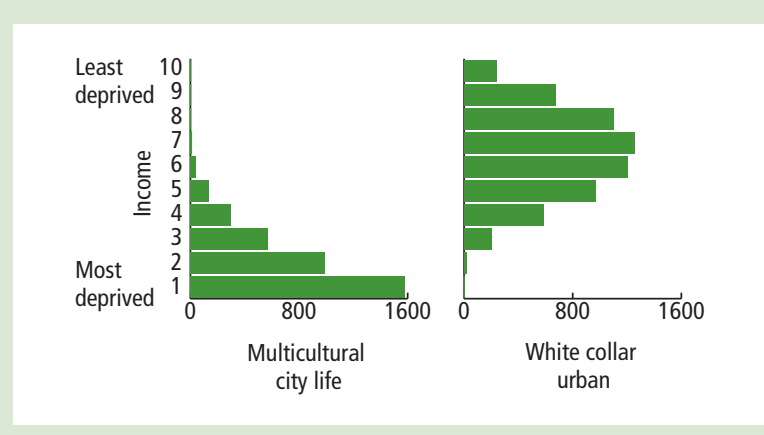

These particular histograms show the profile of the Income deprivation domain for two of the AC Groups.

A graph with a peak at the top (large ranks - so low deprivation) shows a relationship where areas of this type are generally less-deprived in this domain. Equally a peak at the bottom of the graph shows that areas of this type are largely more-deprived. Here we see that Multicultural City Life areas are highly deprived on the income domain (with 99 per cent of the LSOAs falling in the bottom half of the distribution) while White Collar Urban areas are much less deprived (with 72 per cent of the LSOAs falling in the top half).

The value of these multiple graphs is most clearly seen, however, by considering the whole graphic. Essentially each 'column' on the graphic shows the 'profile' of deprivation for those LSOAs which fall within a given Area Classification group. Each 'row' of graphs shows how deprivation of this type varies across the different area types. It is the intersection of these two sets of information, however, that provides most information. Patterns in the distributions provide clues about relationships and, sometimes subtle, differences in the shapes of the distributions reveal much more information than could be obtained from a set of correlations.

Note that in order to ease comparison, as far as possible, common scales have been used for these graphs. The first two columns have a different scale from the rest of the graphs but in all cases graphs within a column have the same scale - so comparisons across the domains remain valid.

Figure 6.14 considered later follows a very similar scheme showing the relationship between the IMD and the National Statistics Rural/Urban Area Classification. 
Figure 6.12 Relationship between the National Statistics Area Classification and the Indices of Deprivation, 2007
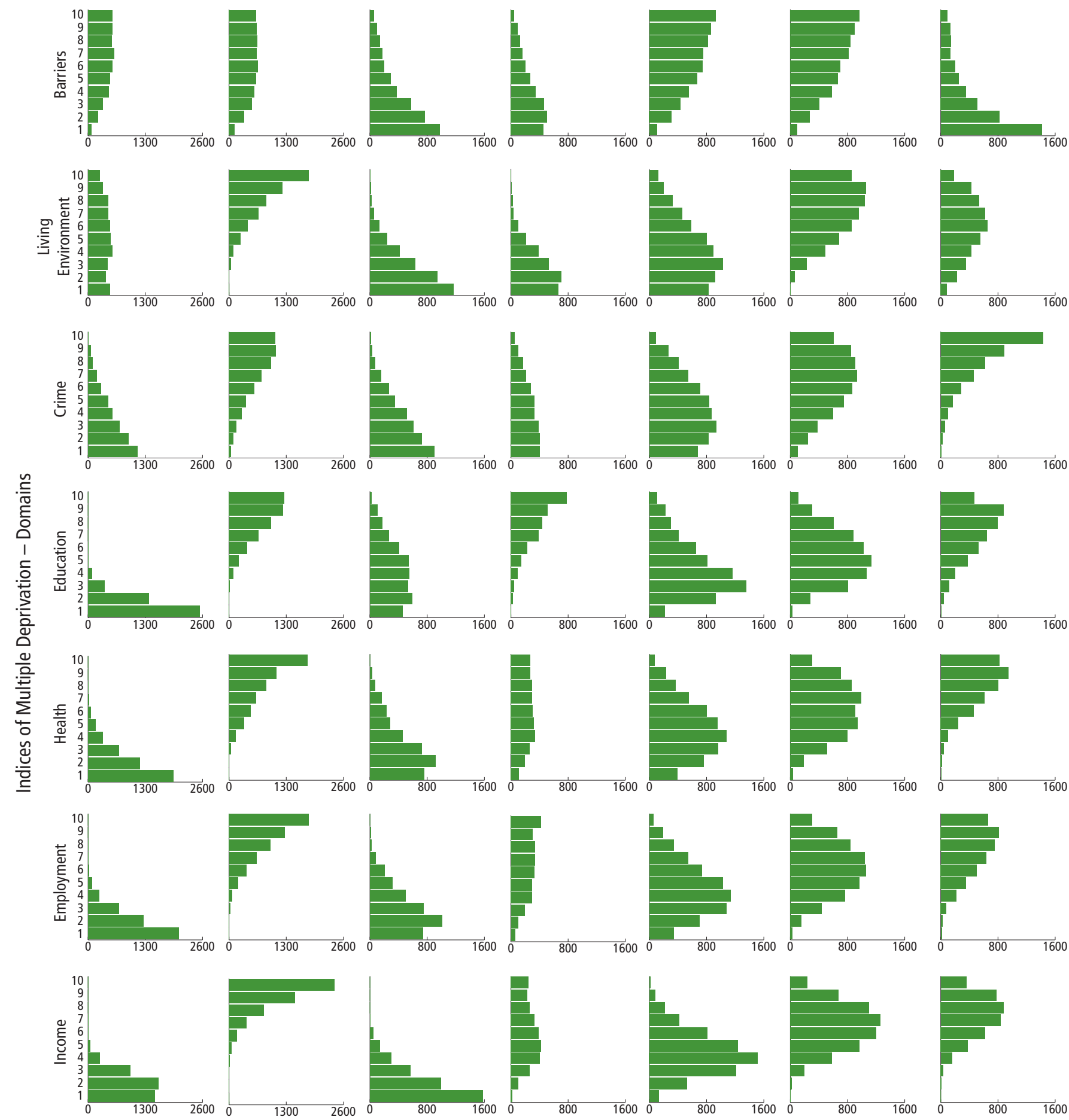

Disadvantaged Urban

Urban Fringe
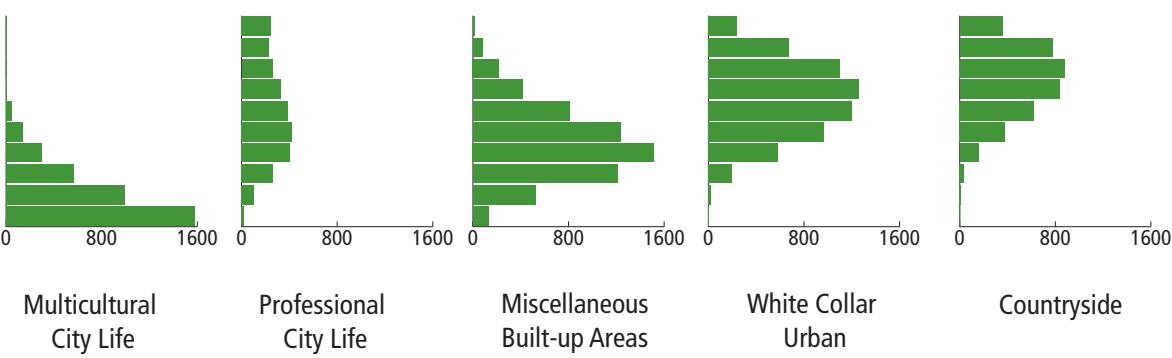
Professional
City Life

\section{Miscellaneous} Built-up Areas

White Collar

Countryside

National Statistics Area Classification
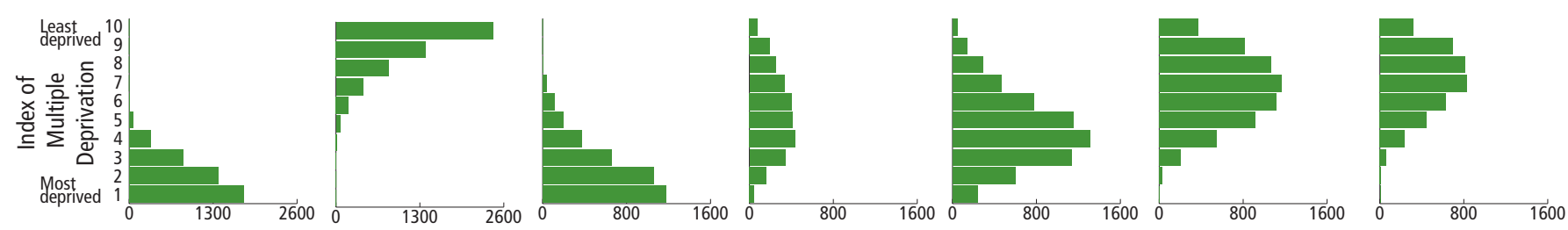


\section{Overall relationship between area types and the IMD}

The bottom row of charts on the page shows how individual groups fare in the overall IMD. What is immediately obvious is that there are strong patterns here. Some groups do well (they have lots of LSOAs in the higher deciles - at the top of the graph) while others do badly (their LSOAs tend to fall in the lower deciles - at the bottom of the graph).

Consider first the Disadvantaged Urban Communities group the graph in the extreme bottom left hand corner. Disadvantaged Urban Communities are the most deprived group overall with 72 per cent of its LSOAs falling in the bottom 20 per cent of the IMD. The Multicultural City Life group (see the third graph in this row) is also highly deprived according to the IMD with 62 per cent of its LSOAs in the bottom 20 per cent. What is most striking here is the strength of the relationship - there are virtually no LSOAs in either of these categories in the top half of the IMD (0.2 per cent and 4 per cent respectively). Very nearly every LSOA of these types is deprived.

The pattern for Urban Fringe is equally strong, but in the opposite direction - only 2 per cent of Urban Fringe LSOAs are in the bottom half of the IMD ranks. Urban Fringe LSOAs, a largely 'suburban' group, are by far the least deprived group overall

Other groups of the Area Classification show less extreme patterns - with most LSOAs falling somewhere in the middle of the IMD ranks. Professional City Life and Miscellaneous Built-up Areas are marginally worse off than White Collar Urban and Countryside areas which have very similar broadly 'lessdeprived' profiles. The White Collar Urban group also tends to occur on the edge of cities.

Note that the 'thinner' graph for Professional City Life is the result of the smaller number of areas categorised in this group - remember that these graphs show counts of LSOAs and that the graphs have (two) common scales.

This initial inspection suggests that the AC Groups have a strong relationship to the IMD. Disadvantaged Urban Communities and Multicultural City Life areas are almost universally highly deprived while Urban Fringe areas are almost never deprived. Other groups fall somewhere in-between.

Clearly the (social) type of area you live in is a strong predictor for how deprived your area is going to be - particularly at the extremes. At first this finding seems obvious and rather facilebut a closer inspection shows such a link is, at least statistically, not inevitable. The Area Classification is built around the social characteristics of areas at Census time 2001. The Indices of Deprivation are largely built around much more recent outcomes - school results, benefits and levels of crime etc. Although there are a few direct statistical links and there will be some clear correlations between related variables the Area Classification and IMD are measuring distinctly different dimensions of the characteristics of areas - so the strength of relationships evident here are valid and interesting.
Relationship between area types and the individual domains

The next step is to look at the individual domains of the IMD and how they relate to the AC Groups.

What is immediately striking here is that the bottom three domain rows (Income, Employment \& Health) are remarkably similar and an almost perfect reflection of the IMD results. This helps confirm the evidence of the similarity of these domains shown on the maps and boxplots included earlier in this article. To some extent this similarity is because these domains are the most highly weighted in the IMD but it is also because these domains are highly correlated with each other.

The other domains of the IOD are less predictable.

Education is similar to the IMD and the domains already discussed but Disadvantaged Urban Communities strongly dominate the poorly performing areas. This group which consists of two sub-groups - 'struggling urban families' and 'blue collar urban families' - makes up 16 per cent of the UK population but accounts for 78 percent of the LSOAs falling in the most deprived decile (10 per cent) on Education. Conversely the other badly deprived group Multicultural City Life, whose definition includes a very significant ethnic population, is much more deprived on Income than any other group, but does relatively well on Education. The most interesting group on Education however is the Professional City Life group. These areas perform extremely well on education this running against their results on the IMD where they are distinctly middling.

Crime shows a similar pattern to the other IMD domains but the differences and contrasts between areas are less marked. Perhaps this is a mirror to the map we looked at earlier. Crime is a problem in the city - but extends beyond it into the suburbs. The strongest pattern here is in the Countryside group which shows very little Crime deprivation. Some 58 per cent of Countryside LSOAs are in the top 20 per cent least deprived in the IMD.

Living Environment and Barriers, the final two domains, provide mixed patterns on the graphs just as they did on the maps.

The 'most deprived' group Disadvantaged Urban Communities is not particularly deprived on either of these domains whereas Multicultural City Life areas and Professional City Life are. This latter group is relatively well educated - but (like the Multicultural group) they live in city centres where the Living Environment and Barriers domains fare badly. The largest proportion of this group are also in London, Birmingham and Manchester where housing affordability (part of the Barriers domain) will also be a problem.

It is interesting to note that Countryside does not rank particularly well on the Living Environment domain - faring worse than White Collar Urban and Urban Fringe areas. This seems counter intuitive; rural areas would usually be considered to have a good environment, green space and good air quality. 
This apparent anomaly however arises from the definition of 'Living Environment'. As well as air quality and road traffic accidents this domain includes measures of housing stock quality and central heating. It is likely that these elements are complicating the picture for Countryside areas (and incidentally improving the performance Disadvantaged Urban

Communities) on this domain.

Most striking in these last two domains, however, is the very poor performance of Countryside areas on the Barriers domain - this is the only domain on which the Countryside group does badly. This will be largely the result of the inclusion of the access to services in Barriers but the affordability of housing may also be playing a role. Miscellaneous Built-up areas and White Collar Urban do best on this domain. These groups are often located on the edge of urban areas - in less expensive housing but with reasonable access to services.

It is also possible to look at this graphic using the opposite dimension - the profile of each loD domain across each area type - and readers may wish to look at this. Only the most obvious patterns are described here and you are likely to be able to spot more subtle differences and relationships throughout the diagram. It is the ability to pick out such subtleties that makes this type of graphic useful for summarising and analysing this type of complex, cross-domain relationships.

Some conclusions will be drawn from this analysis later - after looking at the IMD from another dimension - its relationship with Urban and Rural areas.

\section{Comparison of the Indices and the Rural and Urban Area Classification}

The National Statistics Rural and Urban Area Classification (the RU classification) provides a simple way of summarising how rural (or otherwise) each area in the country is. The classification is built using a complex set of criteria - but it is based strongly upon relative population densities in different areas $^{8}$.

The classification, which covers England and Wales, has been used in developing policy and analysing rural areas and is available at a range of geographic levels. Because we are considering the relationship to the IMD and its domains we will focus here on the classification at the LSOA level and only for England.

The classification makes little distinction within urban areas (which it takes to be areas with more than 10,000 resident population) - but it does provide some subtle distinctions between different types of rural area and so is useful for the type of analysis we want to carry out here.

What is particularly useful about the classification for our purposes is that it is statistically completely independent from the IMD and its domains. The RU classification makes use of a set of boundaries for the edges of urban areas and the distribution of the population in deciding how rural or urban an area is - and nothing else.

Categories of the Rural and Urban Classification and the meaning of 'sparseness"

At the LSOA level the classification consists of six categories:

- Urban $>10 k^{9}$ - Less Sparse

- Town and Fringe - Less Sparse

- Village, Hamlet \& Isolated Dwellings - Less Sparse

- Urban $>10 k-$ Sparse

- Town and Fringe - Sparse

- Village, Hamlet and Isolated Dwellings - Sparse

The groups here are fairly self explanatory - except that the use of 'sparse' and 'less-sparse' needs a little explanation. Sparseness is described in the methodology as the 'context' and has been determined by the fact that these LSOAs have a very small population falling in their wider vicinity - a circle drawn $30 \mathrm{~km}$ around the LSOA concerned. This is a complicated concept but is useful here. Although the term 'remote' is never used in describing the categories, by definition, all sparse areas will be remote. Note however that the converse is not true - not all remote areas are categorised as 'sparse'. A couple of very 'remote' towns which happen to fall within $30 \mathrm{Km}$ each other will not be sparse. Equally, much of Dartmoor, which would fit firmly into most people's definition of 'sparse', ends up as a 'less sparse' due to its proximity to Plymouth. Nonetheless, for the purposes of this article, sparse areas can be considered as remote. This allows us to further test the earlier supposition that there is a relationship between remoteness and deprivation.

The vast majority (81.1 per cent) of the population of England live in areas categorised as Urban (79.9 per cent less-sparse and 0.2 per cent sparse). 9.6 per cent are in the Town or Fringe groups (9.1 per cent less-sparse and 0.5 per cent sparse). 9.3 per cent are in Village, Hamlet and Isolated Dwelling groups (8.6 per cent less-sparse and 0.7 per cent sparse).

It is obvious that the 'sparse' groups are extremely rare - only 1.2 per cent of the population live in 'sparse' SOAs. This is hardly surprising. To become an 'Urban $>10 \mathrm{~K}$ - Sparse' area, for example, a town needs to have a population of more than 10,000 residents - but to be separated from any other

$910 K=10,000$ resident population (Census 2001).
8 Detailed information on the National Statistics Rural / Urban Classification can be accessed via the Neighbourhood Statistics Service website: www.neighbourhood.statistics.gov.uk 
significant settlement. It is obvious that this is going to be extremely rare. Accordingly 98.8 per cent of the population lives in 'less-sparse' areas.

Map 6.13 shows the distribution of these different types of area for England and Wales. Wales is included only for completeness and is not discussed in this article. The pattern here is exactly as one would expect. All of the major conurbations stand out, surrounded by their suburbs. Patterns of development in coastal areas particularly along the South Coast - and even the influence of the radial transport network around London are all easy to spot. One striking feature here, however, is the distribution of the dark green 'Village, Hamlet and Isolated Dwellings-Sparse' group. These 'remote' rural areas show a clear parallel with the areas of relative deprivation identified earlier - in Northumberland, the Welsh borders, around the Wash and across the north of Devon and Cornwall. A comparison of this map with Map 6.3 shows the clear parallel - a confirmation of what was expected - that these areas are the most remote from population.

\section{Comparing the Rural Urban Classification and the IMD}

With a basic understanding of the Rural Urban Classification it is possible now to look in more detail at its relationship to the IMD. The method used here is exactly the same graphical one used earlier with the Area Classification.

Figure 6.14 shows the relationship between the RU

Classification and the IMD and its domains.

See the box before Figure 6.12 for guidance on how to interpret this graphic.

This time the 'columns' show how each area type fares on the different IMD domains. The 'rows' show the profile of deprivation for each IMD domain across the different types of area.

Unfortunately, because the numbers of LSOAs falling into each area type vary so much, it has not been possible to keep the scales consistent across the graphs this time. The scales have, however, been kept consistent within each column - so comparison is valid.

As noted before the 'sparse' groups are extremely rare so they have been grouped together at the right hand side of the diagram - the lighter coloured columns. The small numbers involved mean it is important to take some care in interpreting these particular graphs. Their relative 'roughness' in some cases is the result of natural variation within small numbers which is smoothed out in the 'less-sparse' graphs. The broad pattern the sparse graphs show will be broadly representative - but it is important not to read too much into small variations in the detail.

With these caveats in mind it is possible to start to look at what the RU Classification tells us about deprivation.

\section{Relationship with the overall IMD}

Focussing first on the IMD as a whole (and the first three columns) the patterns seem to bear out our earlier findings. Urban areas are broadly deprived - but show a significant mix of levels of deprivation. Notice that the IMD 'kicks-out' a little in the top decile (as a result of similar patterns in all of the bottom 4 domains). The urban areas contain a small but significant set of LSOAs which are not at all deprived. To some extent this may reflect the common observation of relatively affluent areas in many city centres. Remember however that the RU classification definition of Urban is also a very broad one, containing more than 80 per cent of the population. Map 6.13 shows how far the major conurbations spread out into their 'suburbs' - and so into less deprived or mixed areas perhaps picking up some of these affluent areas here. Urban areas tend to be deprived, but not universally so.

Town and Fringe - which are largely sub-urban and 'market town' areas - do very well and are largely less deprived; 76 per cent of this group fall into the top half of the graph. Village, Hamlet and Isolated Dwellings are broadly non-deprived and have virtually no LSOAs in the most deprived two deciles. They do not, however, do quite as well as the Town and Fringe group at the very top of the distribution. They actually have a lower proportion in the top decile than either of the other two area types.

A look at the 'sparse' groups on the IMD shows a radical difference - all of these groups show a tendency to be mildly deprived. They have very few of the most deprived LSOAs but equally they are unlikely to have many areas in the least deprived.

If anything the urban areas do worst of the three remote (sparse) groups - but as noted before the numbers are too small here to place too much trust in these patterns.

The broad message does seem to be clear however. If you are remote it doesn't matter what type of urban or rural area you are in - you are more likely to be in a deprived area.

\section{Relationship with the individual domains of the IMD}

Considering first the 'Less-Sparse' groups the usual patterns are obvious. Income, Employment, Health and Education domains show strong parallels with the IMD - though there are some differences.

Town and Fringe does well again on all of these domains - but rather less well in these domains than in the IMD. Further up the graphic we can see that it is getting its very good IMD results from very low deprivation on the Crime, Environment and Barriers domains rather than from these lower ones. Equally the 'Villages' group does better on Employment and Health than the overall IMD would suggest- this time the IMD is being pulled down by poor showings on Environment and, particularly, Barriers

A quick look at the Sparse areas for these lower 4 domains shows a repeat of what we saw on the IMD. In every single 


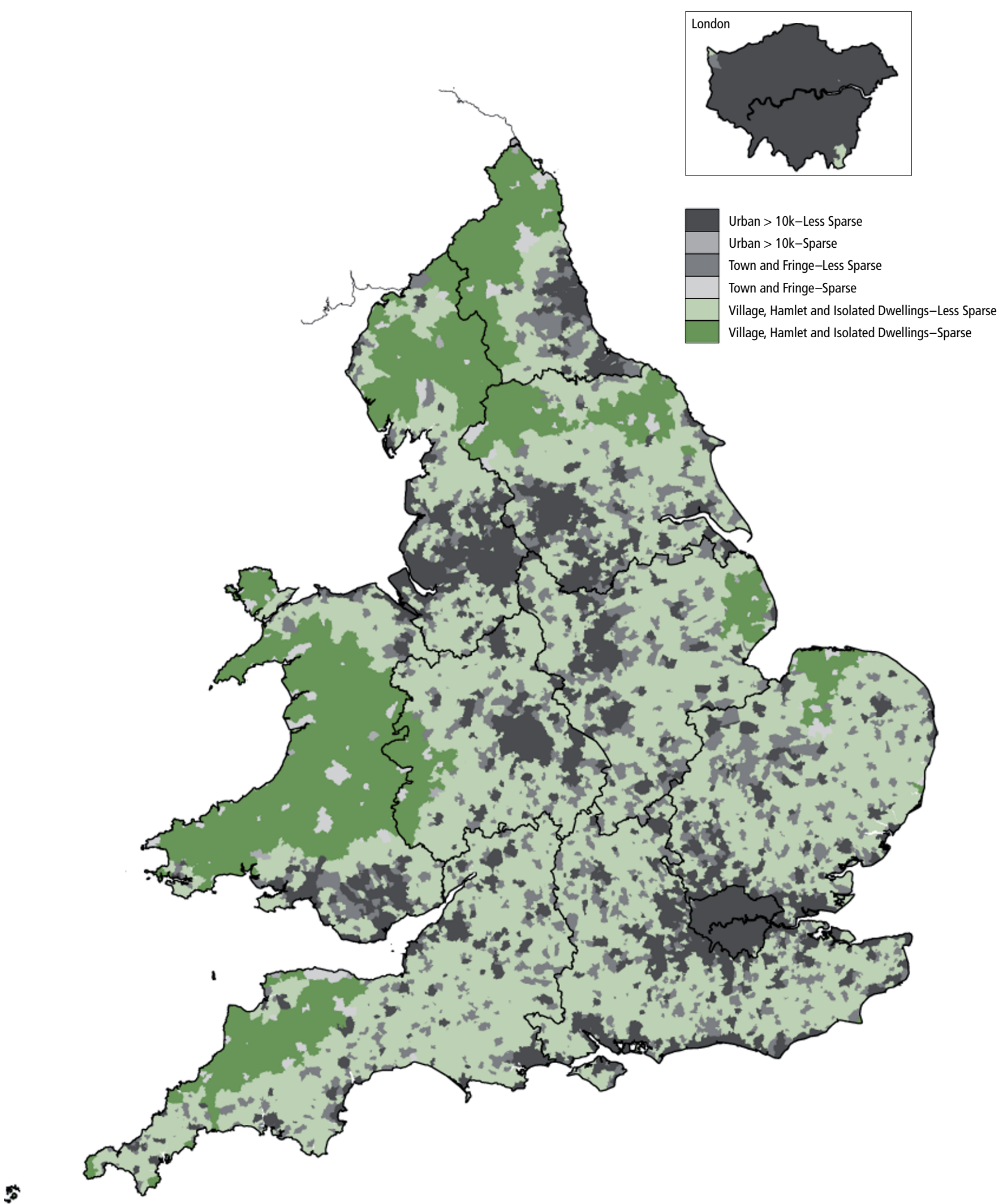


Figure 6.14 Relationship between the National Statistics Rural / Urban Classification and the Indices of Deprivation 2007
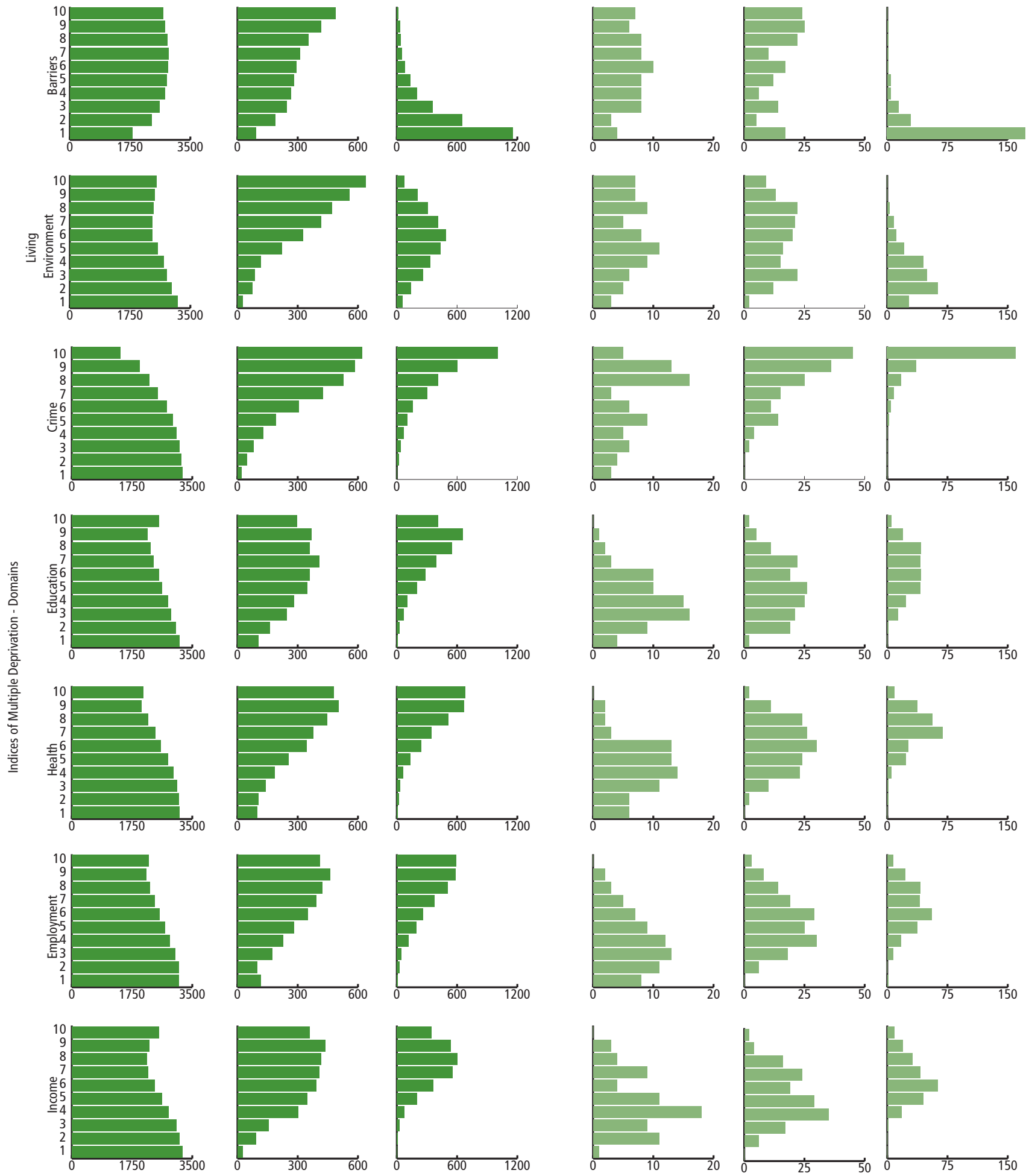

Urban $>10 \mathrm{~K}$

Town and Fringe

Village, Hamlet \&
Isolated Dwellings

Less Sparse
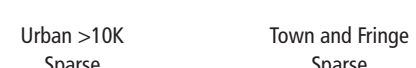

Village, Hamlet \& Isolated Dwellings

National Statistics Rural-Urban Classification
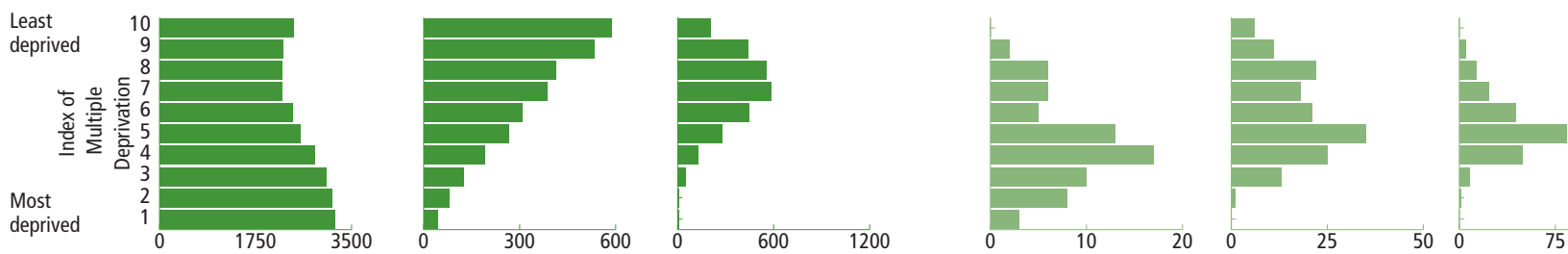
case the profiles are worse in Sparse areas than in the respective Less Sparse areas. Again the remote areas are more deprived.

Crime is different. As we saw before Crime clearly has a strong Urban/Rural dimension. This is the domain where Urban areas do worst of all - and 'Villages' best of all. In this case the Sparse (remote) areas do better than their respective Less Sparse areas. As the maps suggested earlier the more remote the better when it comes to avoiding crime deprivation.

As ever the Living Environment and Barriers domains produce mixed, and sometimes conflicting, results. Urban areas have mixed results on both domains, Town and Fringe do fairly well on Barriers - and extremely well on Living Environment (its best result). The 'Village' group is particularly interesting on Environment - a distinctly middling result and a poor one compared to its results on most other domains. It seems likely that this reflects poor results on the indoors (housing and central heating) indicators balancing out good results on the outdoors (air quality and traffic). Equally 'Villages' produces the predictable very highly deprived performance on the Barriers domain - pulled down by poor scores on access to services. Note that results for Sparse Villages are predictably more extreme still.

As we have seen in the earlier discussion Barriers in particular seems to produce results that run contrary to the scores on the other domains.

In the case of 'Village' areas- the very poor showing on Barriers is mirrored by a very good showing on Crime. Further inspection of the data shows that the extreme values in both cases are in roughly the same areas so these results largely cancel each other out in the IMD (since their weightings are the same).

This does not, however, mean that there is any flaw in the logic of the IMD. It is actually only in the more rural areas that this cancelling out occurs. This confirms the common observation that there are up-sides and down-sides to living in rural areas. Whether an individual living in a remote rural LSOA actually considers their location to be deprived due to such factors requires a more subjective interpretation. Some people relocate to rural areas precisely because such areas are isolated, and do not consider such geographical barriers to have a negative impact upon their daily lives.

Again this has been only a summary analysis - the reader may want to study these graphs further and look for the more subtle patterns.

\section{Conclusions}

Using the IMD for small area analysis.

The first conclusion to be drawn here is that in order to get any real understanding of spatial patterns of deprivation it is important to use data at as low a level as possible - and this means at the LSOA level. Using district level summary measures for either the IMD or Area Classification would largely mask some of the subtle effects identified here.

These studies have also shown that different types of areas perform very differently on individual domains. What is very obvious, however, is that in carrying out any type of analysis the user needs to be very aware of the complexity and summary nature of the IMD measure. Often consideration of the individual domains or even sub-domains will offer more insight than just using the IMD. Equally it is important that users are aware of some of the sometimes conflicting characteristics of the different domains.

The influence of the Crime, Environment and Barriers domains do complicate the picture - but notice that they do this in a very specific way - relating strongly to the rural-ness of the area. The Barriers domain which is frequently dominated by accessibility does often run counter to the results in the other domains but this does not make its contribution to the IMD wrong or unnecessary. The inclusion of some element of accessibility in the IMD does seem entirely appropriate - it just happens to be important in areas which are otherwise relatively less deprived.

It is of course possible to argue with individual elements of the IMD or to take different views of what the relative weightings should be. Some of the domains contain apparently conflicting pulls on the scores. Again however this is not evidence that there is a weakness in the logic of the measure. Different areas do suffer different types of deprivation. We just need to be aware of these in drawing conclusions.

It is fairly obvious that this is a difficult area and that assessment of multiple deprivation is inevitably partially subjective. This study has not looked to test the validity or otherwise of the IMD as a measure. What is has shown however is that the IMD as currently formed reveals strong and apparently consistent relationships between location, area type and deprivation.

This does not, in itself, validate the success or otherwise of the IMD in identifying deprived areas but it does at least show that it is a consistent measure.

\section{The influence of "place" on deprivation}

This article has done much to confirm observations that may seem obvious - but it has also uncovered some subtleties in the relationship between the domains of deprivation and different types of places.

It has proved that the domains of the IMD have very distinct and, in some cases, quite different patterns. It is impossible to prove any causal relationship with this type of analysis but the strength of the patterns revealed on the graphs clearly shows that different types of places do suffer very different fates in terms of deprivation.

The indication is that geographical 'extremes' help contribute to deprivation - cities and remote areas are most likely to be 
deprived - though their deprivation will often be driven by very different factors.

Throughout all of these brief studies we have seen the same areas performing best - the 'Town and Fringe' in the Rural Urban Classification; the 'Urban Fringe' in the Area

Classification; the suburban areas to the main cities on the maps. The next most suburban of the Area Classification groups, White Collar Urban, also does well. All of the evidence here suggests that areas near the city - but not in it - are almost always the least deprived.

The study has also confirmed what one would expect - that Urban areas tend to be the most deprived - but also that the pattern here is far from universal. Urban areas can also be relatively very well-off particularly on specific domains and for specific groups. The broad definition of 'Urban' used here tends to cover a wide range of types of areas and it may be appropriate to use the Area Classification to supplement any analysis of differences within the urban areas.
Rural areas perform relatively well on all measures (except Barriers) and do particularly well on Crime but they do not perform as well as suburban areas. The most remote areas fare much worse - often displaying some of the highest levels of deprivation.

This article has revealed a number of ways in which place influences deprivation and shown that deprivation is driven by a complex, sometimes conflicting, set of factors.

While these factors lead to subtle variations in different domains and in different types of areas one overall pattern emerges. Geographic extremes - the inner city and the most remote rural areas - coincide with extremes of deprivation while suburban and 'middle-England' almost entirely escape them.

\section{Office for} National Statistics

\section{Related titles from the Office for National Statistics}

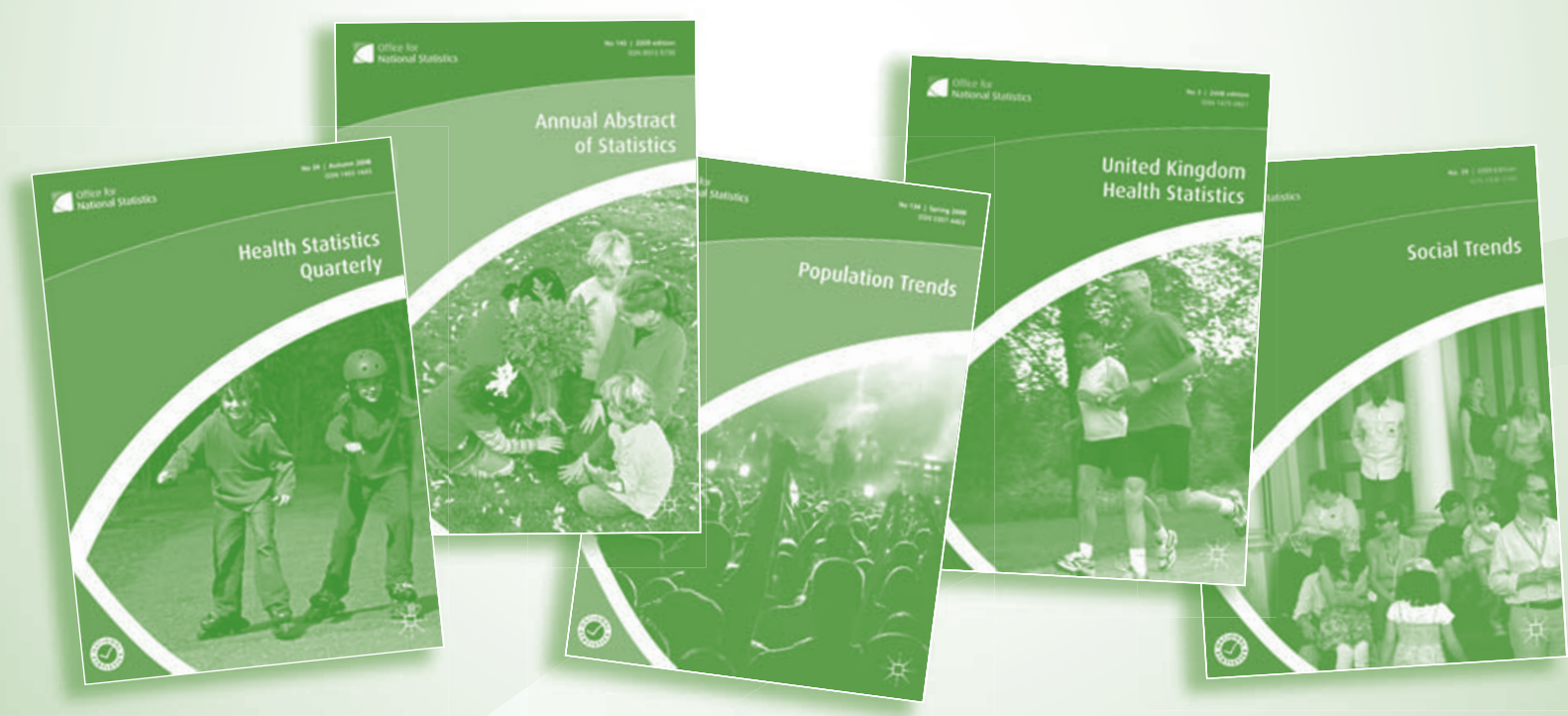

www.palgrave.com/ons/ 